\title{
Interactive comment on "A full Stokes subgrid model for simulation of grounding line migration in ice sheets using Elmer/ICE(v8.3)" by Gong Cheng et al.
}

\section{Anonymous Referee \#3}

Received and published: 4 November 2019

This paper presents a subgrid interpolation across the grounding line for the Stokes equation. This interpolation is based on the stress balance at the base of the ice, $\chi=p_{w}+\sigma_{n n}$ with $\sigma_{n n}$ the normal deviatoric stress at the ice base, and $p_{w}$ the water pressure. If the last grounded element is at node $i$, and the first floating element is at $i+1$, then the position $x_{G L}$ of the grounding line is determined as the first point where $\chi$ goes to zero. This position is then used in the evaluation of the weak form of the basal boundary condition.

Unfortunately, the incorrect citation style and at times awkward writing of the paper (in particular the introduction) distract from its contents. These need to be corrected 
before publication can be considered. Scientifically, the approach is a logical first step for interpolation of the basal boundary condition across the grounding line, and the same approach has been used in Seroussi et al. (2004), though not for a Full Stokes model. That said, I think that the analysis of the results could be improved significantly by

1. comparison with other interpolation schemes in 1 horizontal dimension, and

2. extension to 2 horizontal dimensions. The latter case is briefly discussed in lines 229-333, but I think an implementation would show whether this approach in indeed able to deal with complex grounding line geometries.

These kinds of comparisons are standard for the study of numerical grounding line schemes (see e.g., Seroussi et al., 2004, Feldmann et al., 2014).

For point 1 above, I am specifically interested in seeing a comparison to an interpolation of the basal shear stress constant $\beta$ between the last grounded and the first floating point, i.e., if one would multiply $\beta$ with $\left(x_{G L}-x_{i}\right) /\left(x_{i+1}-x_{i}\right)$, how would the results differ? This is the interpolation scheme traditionally used in depth-integrated models (e.g., Feldmann et al., 2014, Pattyn et al., 2006) and also introduced as SEP1 in Seroussi et al. (2014). I am wondering whether such an interpolation alone would already provide the observed improvement in the numerical performance, as suggested from my reading of Seroussi et al. (2014). I am also sceptical about the effect of setting $\beta$ to $\beta / 2$ in the interpolated cell, as suggested in lines 282-283? This introduces an additional interpolation which is similar to the interpolations used in models with a structured grid, and has no physical basis in the presented scheme as the interpolation is already done by splitting up the integral for the boundary condition.

Moreover, it wasn't completely clear to me which parts of the FEM implementation in Elmer/lce were actually altered. For example, my impression of the time-stepping

Printer-friendly version

Discussion paper 
scheme is that it is basically the same as in Durand et al. (2009a), in which case section 3.3. is unnecessary.

Other comments (kept short as I think the entire paper needs to be rewritten):

- Better use $\dot{\varepsilon}$ for the strain rate, $\tau$ is usually used for a stress tensor

- Equations (7) and (8) are the kinematic boundary conditions, and should be referred to as such

Interactive comment on Geosci. Model Dev. Discuss., https://doi.org/10.5194/gmd-2019-244, 2019. 